\title{
Le territoire d'État entre imposition et subversion : exemples saharo-sahéliens
}

Pierre Robert Baduel

\section{(2) OpenEdition \\ 12 Journals}

Édition électronique

URL : http://journals.openedition.org/conflits/230

DOI : $10.4000 /$ conflits.230

ISSN : $1777-5345$

Éditeur :

CCLS - Centre d'études sur les conflits lilberté et sécurité, L'Harmattan

Édition imprimée

Date de publication : 15 mai 1996

ISSN : 1157-996X

Référence électronique

Pierre Robert Baduel, « Le territoire d'État entre imposition et subversion : exemples saharo-

sahéliens », Cultures \& Conflits [En ligne], 21-22 | printemps-été 1996, mis en ligne le 15 mars 2006, consulté le 30 mars 2021. URL : http://journals.openedition.org/conflits/230 ; DOI : https://doi.org/ $10.4000 /$ conflits.230

Ce document a été généré automatiquement le 30 mars 2021.

Creative Commons License 


\title{
Le territoire d'État entre imposition et subversion : exemples saharo- sahéliens
}

\author{
Pierre Robert Baduel
}

1 Tandis que la crise du Moyen-Orient d'août 1990 à février 1991 montait inexorablement aux extrêmes, les leaders politiques occidentaux déclaraient l'avénement d'un "nouvel ordre mondial" ou d'un "nouvel ordre international" assurant la primauté du droit sur la force ${ }^{1} .1989$ avait ouvert la voie avec des changements politiques de taille : la chute du mur de Berlin et des régimes communistes de l'Europe de l'Est. 1990 connaitrait, parallèlement à l'explosion de la crise moyen-orientale, plus positivement la réunification de l'Allemagne ${ }^{2}$, et du Yémen ${ }^{3}$. Puis ce fut avec la fin de l'apartheid, l'avènement inespéré de la démocratie en Afrique du Sud. Mais ce "nouvel ordre mondial" dont Gorbatchev fut un des hérauts n'avait pas programmé la disparition de l'URSS (août 1991) et son cortège de bouleversements proches et plus lointains, et s'il semblait promettre enfin à l'ONU, une centralité internationale effective qui la pousserait en 1992 à intervenir spectaculairement en Somalie (J.-M. Sorel 1992), cette organisation allait montrer rapidement son impuissance en ex-Yougoslavie, se déconsidérer au Rwanda (1994) et essuyer au total l'échec cuisant de l'opération Restore Hope (retrait total des Casques bleus en mars 1995). Des Etats aujourd'hui en crise comme l'Algérie ou l'Inde, à plus forte raison l'ex-Yougoslavie, seraient-ils en fait orphelins de l'ancien ordre international qu'ils étaient parfaitement arrivés à instrumentaliser alors qu'aujourd'hui, nus et crus, ils ne peuvent plus sublimer leur faiblesse, comme le prétend Zaki Laïdi ${ }^{5}(1994,41)$ ? Dans nombre de ces événements en tout cas, la question du rapport au territoire occupe une position centrale, qu'il s'agisse de réunifications, de séparations (Tchécoslovaquie), de conquêtes (guerres du Golfe) ou encore de mouvements nationalitaires (de la revendication berbère en Algérie à la revendication sécessionniste kurde), pouvant conduire de déplacements ethniques (Erythrée à l'égard des Ethiopiens) à l'ethnocide méthodique (Bosnie, Rwanda). L'onde de choc du "nouvel ordre international" en tout cas se propage. Et le continent africain, si oublié par ailleurs, pour une fois, est pleinement dans la course ... L'“ancien régime 
international" africain remis en cause. Ce qu'on pourrait appeler l'“ancien régime international" du continent africain, dessiné lors de la conférence d'arbitrage-partage des puissances européennes convoquée par Bismarck à Berlin en 1884-1885, et coulé dans le bronze des "tables de la loi" onusiennes des libérations nationales, a fortement modelé l'organisation régionale et interne des Etats de ce continent. Et d'abord la formation des Etats-territoires. La clôture des espaces étatiques africains, faut-il le rappeler, n'intervint effectivement pour l'essentiel que comme effet de la course à l'empire que se livraient sur ce continent quelques puissances européennes : avec leur expansion, qui mettait un terme à l'existence (de leur point de vue) de toute terra nullius, on parvenait au temps d'un monde politiquement fini ou plein ${ }^{6}(\mathrm{P}$. R. Baduel 1984). Non qu'il n'existât antérieurement des espaces délimités : les communautés élémentaires (ethnies, tribus) savaient globalement l'ordre politique dont elles relevaient, qu'il s'agît des émirats ou chefferies de l'actuelle Mauritanie, des royaumes soudanais ou encore, par exemple, de l'émirat touareg de l'Ayar (nord-ouest de l'actuel Niger), même si elles cherchaient parfois à s'y soustraire, surtout dans les moments où les colonnes fiscales étaient annoncées : leur évitement n'était cependant possible que parce qu'existaient encore des territoires sinon "vides" du moins mal contrôlables ${ }^{7}$. Mais en dehors de quelques régions (ainsi pour le Maghreb dans la partie septentrionale du Maroc, de l'Algérie ou de la Tunisie), la frontiérisation entendue comme clotûre de l'espace étatique, ligne de contrôle du mouvement des gens et des marchandises, n'intervint complètement qu'avec l'inscription de ces régions dans l'ordre international européen, puis mondial. Mais les frontières actuelles qui, dans l'empire français d'Afrique, résultaient pour l'essentiel des délimitations territoriales nées des besoins de l'administration des colonies et protectorats 8 (A. Martel 1965, S. Sayagh 1982, O. Vergniot, 1986), ont pu être contestées par les États nouvellement indépendants, parfois en recourant aux armes : ainsi entre le Maroc et l'Algérie pour la frontière saharienne dès l'indépendance de celle-ci et bien avant la question de l'exSahara espagnol, entre le Maroc et la Mauritanie (dont le Maroc niait jusqu'au droit à l'existence", (par exemple Ch. Serghini, 1982), entre la Libye et le Tchad ${ }^{10}$ (B. Lannes 1982, A. Martel 1992), ou encore entre le Sénégal, le Mali et la Mauritanie (P.R. Baduel 1990) ${ }^{11}$. Les failles, les craquèlements, puis l'effondrement de cet "ancien régime international" ont libéré d'autres jeux politiques, en fait souvent initiés depuis un plus ou moins grand nombre d'années, mais désormais au cours plus aléatoire. Or l'un des grands principes de l'OUA qui avait structuré cet "ancien régime international" africain, à savoir l'affirmation de l'uti possidetis et de l'“intangibilité des frontières héritées de la colonisation"12 (P.R. Baduel 1985) au nom duquel avaient été rejetées quasi unanimement les revendications katangaise, biafraise, érythréenne, ogadenaise, etc $^{13}$ (M.-Ch. Aquarone 1987), a connu récemment une importante entorse, non plus du fait d'un coup de force comme au Sahara occidental ${ }^{14}{ }^{15}$, mais le plus légalement du monde. Cette brèche fut ouverte en 1993, presque en catimini, par l'accession à l'indépendance de l'Erythrée, ex-province de l'État abritant depuis sa création le siège de l'OUA elle-même ${ }^{16}$ (R. Marchand 1993). Etait mis en même temps terme à un dogme de l'OUA: colonisation n'était plus seulement synomyme d'Occident, un État authentiquement africain pouvait opprimer un peuple lui aussi africain et celui-ci pouvait vouloir légitimement s'en libérer ${ }^{17}$ ). L'uti possidetis ne devait plus être opposé systématiquement au principe de "libre détermination des peuples"18: une leçon à méditer, par exemple par le Soudan. Alors que, pour des raisons qu'illustra dramatiquement le Rwanda et sur lesquelles je vais revenir à partir d'autres cas, on 
aurait pu s'attendre dans ce cas de figure à l'amorce d'une nouvelle approche du rapport de l'État au territoire en terre africaine, on est plutôt devant une nouvelle avancèe de l'État-nation-territoire? D'autres conflits africains, durs bien que de moindre notoriété médiatique, continuent encore sur une bande qui va de la Corne de l'Afrique à l'embouchure du fleuve Sénégal : la question du Somaliland, liée à l'affaire somalienne ${ }^{19}$; la rébellion tigréenne; la guerre nord-sud au Soudan ${ }^{20}$ (Ch. Delmet 1994); la crise intérieure tchadienne et la question tchado-libyenne; la rébellion / résistance ${ }^{21}$ touarègue au Mali et au Niger ; et enfin la question ethno-nationaliste en Mauritanie et la crise mauritano-sénégalaise. Ces crises résultent certes au premier abord, de contradictions internes mais celles-ci, sont elles-mêmes pour une bonne part, le produit d'une double surdétermination internationale . En effet si tous ces États sont, dans leur matérialité ethno-territoriale actuelle, des produits de l'ordre international colonial et postcolonial aux effets durables, on constate qu'ils sont tous situés sur ce que je qualifierai, après J.-F. Bayart ${ }^{22}$, de "zone de tectonique des plaques civilisationnelles", où réémerge désormais librement un acteur, le monde arabe, qui régionalement joue un rôle concurrent des grandes puissances et brandit un système de valeur alternatif à l'Occident: l'islam, la langue, voire le nationalisme arabe ${ }^{23}$ (R. Otayek, 1994). Sans aller complètement dans le sens de P. Hassner ${ }^{24}$ qui, à la suite de Kenneth N. Waltz ${ }^{25}(1959,12-14)$ distingue trois "directions" d'analyse des guerres : le problème anthropologique, la nature des régimes politiques et la structure du milieu international, pour bien saisir l'évolution locale de la problématique de la statoterritorialité, je croiserai dans l'étude des conflits saharo-sahéliens, facteurs locaux de différents niveaux, du communautaire à l'étatique, et des facteurs internationaux à différentes échelles, régionale à mondiale. Ne pouvant dans les limites de cette communication procéder à une analyse de toutes ces crises, je limiterai ma réflexion à quelques États saharo-sahéliens contigus dans l'espace (Niger, Mali, Mauritanie, Sénégal) et proches par un certain nombre de caractéristiques qui faciliteront la comparaison. En premier lieu, la configuration interne des communautés y est assez semblable: nous avons affaire à des sociétés hiérarchiques ${ }^{26}$. Ensuite les ethnonationalismes qui s'affrontent mobilisent de part et d'autre les mêmes référents: l'arabisme ou l'arabo-berbérisme contre les négro-africanismes. Les acteurs internationaux interpellés sont, à quelques variantes près, les mêmes : Libye, Algérie, France. Et enfin ces crises sont toutes contemporaines entre elles: si elles ont commencé dés les premières années des indépendances et sont restées depuis endémiques, avec toutefois quelques éruptions de durée et d'ampleur limitées, elles ont connu leur paroxysme à la fin des années 1980 ou au début des années 1990. Mais ces crises présentent un certain nombre de différences, ont fonctionné à fronts renversés. On a assisté à une révolte des Touareg au Niger, des Touareg et Maures au Mali, groupes minoritaires dans ces pays, contre les négro-africains, majoritaires et dénoncés comme monopolisateurs du pouvoir d'État et de ses allocations. En Mauritanie à l'inverse, ce sont les négro-africains soudanophones, minoritaires, qui se sont révoltés contre les Maures majoritaires pour le même motif que les Touareg à l'égard des détenteurs du pouvoir central, allant même jusqu'à accuser les Maures de pratiquer un véritable apartheid ${ }^{27}$, pour ultérieurement se livrer à ce qu'on appellerait en Bosnie une "purification ethnique". Je vais essayer de développer chacun de ces points en analysant les éléments de crise, puis les éléments de solution, le tout tournant autour de la question de la stato-territorialité et de son devenir. Analyse comparée des crises ou la stato-territorialité entre impasses ethniques et ordre international. Disons 
d'abord que chacun de ces pays regroupe dans ses frontières de nombreuses ethnies qui, presque toutes, rayonnent plus ou moins largement au delà de leurs enclosures étatiques respectives. Si les Maures, population arabophone composée aujourd'hui d'arabo-berbères et d'anciens dépendants noirs arabisés, vivent pour l'essentiel en Mauritanie, une fraction minoritaire (environ 50000 ?) est installée de façon ancienne au Mali et une autre l'était plus ou moins anciennement aussi au Sénégal (au moment de la crise de 1989 on avançait du côté du pouvoir de Nouakchott - polémiquement surévalué- le chiffre de plus de 300 000). Mais en Mauritanie vivent aussi des négroafricains soudanophones (20 à $40 \%$ selon les camps consultés) appartenant à des communautés : Soninkés, Haalpularen, Woloffs..., qu'on retrouve des deux côtés de la frontière méridionale, et surtout au Sénégal et au Mali. Les Touareg - dont la rébellion / résistance a retenu l'attention des médias internationaux au début des années 90 sont quant à eux dispersés entre plusieurs États : l'essentiel au Niger (750 000) et au Mali (300 000), beaucoup plus faiblement au Burkina-Faso, en Algérie et en Libye (autour de 50000 pour ces trois pays réunis). Ces statistiques officielles ${ }^{28}$ sont contestées par les Touareg, qui - sur la base de sentiments et non de recensement effectif, comme ils le reconnaissent eux-mêmes, n'hésitent pas à avancer des scores très supérieurs : pour le seul Niger, le Programme-cadre de la Résistance touareg ${ }^{29}$ estime le nombre des Touareg à près de la moitié, voire plus, de la population totale de 7,2 $\mathrm{M}$ d'habitants, ce qui en ferait le "premier peuple" du pays! Outre les Touareg, on trouve au Niger des Peuls, Haoussas, Kanouris, Zarmas-Songhays, Toubous, Gourmantchés ${ }^{30}$ (A. Salifou, 1993, 141). De même au Mali, avec les Bambaras, Mandingues, Soninkés, Peuls, Senoufos, Dogons ${ }^{31}$ Si Moktar ould Daddah, le premier président de la République de Mauritanie, appelait de ses vœux la naissance d'une Nation mauritanienne qui eût à terme transcendé les clivages ethniques, c'est bien, comme le dit l'ancien président du Haut-Conseil de la République qui, comme parlement, assura de 1991 à 1993 la transition démocratique au Niger, A. Salifou, que l'État dans ces régions a précédé plus ou moins artificiellement la Nation, érigeant dès lors "au rang d'un terrible défi la réalisation de l'unité nationale" $32(1993,197)$. Le tableau ethniste que je viens de dessiner rapidement et les remarques du dernier paragraghe pourraient induire l'idée d'une simple juxtaposition des ethnies et donc d'une étanchéité des communautés entre elles. Les observateurs les plus avisés font remarquer, si on prend par exemple comme indicateur les alliances matrimoniales, que la pureté ethnique n'était pas aussi assurée que les Capulets et Montaigus locaux cherchent à l'accréditer pour les besoins de mobilisation de leur cause. Charles C. Stewart ${ }^{33}(1990,167-168)$ rappelle que s'ils n'ont pas été fréquents, les mariages entre Maures et Noirs soudanophones existaient bel et bien, certains personnages politiques d'aujourd'hui illustrant d'ailleurs cette mixité. Dans les zones urbaines nigériennes très cosmopolites, en particulier aujourd'hui du fait de l'exode rural, A. Salifou relève aussi ces échanges "entraînant des métissages à la fois biologiques et culturels que nul ne peut ignorer aujourd'hui" ${ }^{34}$. En fait les pouvoirs autoritaires contemporains, incapables de développer une vie démocratique mais ayant besoin d'une forme de légitimité populaire, ont déployé des pratiques clientélistes de groupes qui ont renforcé le plus souvent les clivages ethniques, voire constitué des forteresses ethniques, dont la logique extrême a été illustrée par la tragique affaire rwandaise de l'été 1994. L'ethnisme en Afrique comme ailleurs ${ }^{35}$, voir par exemple le cas de l'Asie centrale est plus, dans sa configuration actuelle, un produit de l'État lui-même qu'une réalité éternelle et substantielle ${ }^{36}$. D'une certaine manière le refus de la démocratie ne pouvait conduire ici comme ailleurs qu'au 
choix alternatif commode de l'ethnisme, masqué la plupart du temps par des proclamations idéologiques ambitieuses, prétendument universelles, d'autant plus tonitruantes qu'elles étaient creuses. L'ethnisme eut eu cependant des chances d'étanchéité s'il se fut développé sur des territoires relativement distincts. Or la réalité de la répartition territoriale ethnique rend la situation sociale plus complexe, en particulier du fait que dans ces zones d'économie traditionnelle une part essentielle revenait au nomadisme et que l'aire de déplacement des nomades traversait de nombreux espaces de sédentaires ou d'autres nomades (mais ne pratiquant pas le même élevage) relevant d'autres ethnies. D'où par exemple la grande dispersion actuelle des Touareg entre cinq États : Niger, Mali, Algérie, Libye, Burkina-Fasso. Au point qu'un territoire pouvait être l'objet d'usages diversifiés correspondant à des jouissances ethniques différenciées, voire complémentaires. Ce cas de figure se présentait également en Afrique du Nord, par exemple pour les Chaanba qui dans l'immense Sahara croisaient à l'est les Ghribs du Sud-tunisien ${ }^{37}$, ou encore des Merazigues du même Sud-Tunisien dont l'amplitude des déplacements allait des zones sahariennes contrôlées par les Touareg - qui devenaient leurs "protecteurs" - aux portes de Kairouan ${ }^{38}$. Dans ces régions nous étions donc dans ce que j'appelerai des espaces d'interpénétration stratifiée et non des espaces d'exclusivité et d'homogénéité comme tendent à l'être les espaces étatiques modernes. Le cas des populations de la Mauritanie actuelle est à cet égard particulièrement édifiant. L'une des causes majeures du conflit ethnique intra-mauritanien et mauritano-sénégalais porte très précisément sur le rapport des communautés au territoire et ce parce qu'on pourrait dire que, par comparaison avec l'âge précolonial et même colonial, le statut du territoire a aujourd'hui profondément évolué. Dans sa causalité immédiate, le conflit qui a mis la Mauritanie à feu et à sang en 1989-1990, et dont les séquelles perdurent aujourd'hui puisque entre 50000 et 70000 réfugiés mauritaniens soudanophones se trouvent toujours (mars 1995) dans des camps au Sénégal - s'est accompagné de la contestation par les Maures au pouvoir de la mauritanité des soudanophones installés le long de la vallée du fleuve Sénégal. Dans la phase d'aménagements lourds des terres avoisinantes auxquels l'État a procédé depuis quelques décennies, face à la concurrence des Maures, les soudanophones réclament la reconnaissance de leurs droits fonciers coutumiers. Or si les soudanophones, éleveurs (comme les Peuls) ou agriculteurs, sont effectivement concentrés aujourd'hui sur les rives septentrionales du fleuve (et de là se sont ultérieurement dispersés dans toute la Mauritanie urbaine), cette installation pour remonter très haut dans les siècles, ne fut pas constante, voire même reflua du XVIIe jusqu'au XIXe siècle sous l'effet de la poussée méridionale des émirats maures, pour fuir les prélèvements matériels et humains que ceux-ci effectuaient sur eux. Ils repassèrent même le fleuve pour ne le retraverser en sens inverse et massivement - voire encore plus récemment, montèrent ( les Peuls) nomadiser encore beaucoup plus septentrionalement, en accord de pâturage avec les populations maures locales qu'avec la progression de la pénétration française au Sénégal et au Bilad-es-Soudan, le pays des noirs, l'actuel Mali ${ }^{39}(\mathrm{Cl}$. Sentoir 1993, O. Leservoisier 1994). Durant toute la période coloniale, la gestion des populations rurales soudanophones comme maures (pour le nomadisme camelin) permit leur mouvement des deux côtés du fleuve et, les indépendances acquises, il ne fut pas mis un terme à ces pratiques, si bien que des soudanophones mauritaniens continuaient à passer régulièrement le fleuve pour cultiver la rive gauche et inversement, et que des nomades maures pouvaient du Hodh (sud-est) passer au Mali et descendre jusque vers le fleuve Niger, en quête de pâturages 
en période difficile, suivant ces axes méridiens rappelés par Denis Retaillé ${ }^{40}$ (1989). Sur ces espaces économiques, Maures et soudanophones n'étaient pas nécessairement concurrents, dès lors que leur interpénétration spatiale était ici aussi stratifiée ${ }^{41} \mathrm{On}$ peut donc remarquer que même au lendemain de l'Indépendance des pays riverains $\mathrm{du}$ fleuve Sénégal, si la frontière était rappelée (voire rectifiée entre le Mali et la Mauritanie, ou contestée avec la Mauritanie par le président Senghor), celle-ci était admise tout à fait officiellement comme poreuse : "Le territoire civil de ces populations ouest-sahéliennes ne recouvrait donc pas exactement les territoires d'Etat, comme si alors l'opposition entre "droit du premier occupant" (négro-africains) et "droit de conquête" (Maures), à défaut de pouvoir être tranchée, ne paraissait pas dirimante.." Or deux séries de faits allaient modifier totalement le rapport au territoire, destabiliser ces usages sociaux stratifiés des espaces internes et transfrontaliers. D'abord la mise en valeur du fleuve : celle-ci devait transformer des terres de culture de décrue (waalo), donc d'économie précaire, d'économie de la faim, en terres de culture de rente, ce qui ajoutait désormais une plus-value considérable à ce bien foncier et le transformait en produit marchand comme un autre, là où n'existait pour l'essentiel ancestralement qu'une propriété et une jouissance collectives. Si bien que ce qui était marges de la Mauritanie abandonnées aux populations négro-africaines, devenait économiquement central et était intégré dans le jeu de l'économie nationale, détenue elle de façon dominante par les Maures, surtout ceux de l'Adrar, au nord ${ }^{42}$. De leur côté le Sénégal et le Mali, qui participaient à l'opération commune d'aménagement du fleuve, connaissaient les mêmes mutations économiques dans leurs régions, ce qui devait modifier le mouvement traditionnel des échanges humains et matériels entre Maures, Maliens et Sénégalais, mais aussi à bien des égards entre soudanophones des deux rives. Cette mutation de la valeur foncière de ces terres périphériques allait être accentuée du fait d'un second événement tout à fait impossible à maîtriser, la sécheresse qui devait sévir très durement et longuement dans les années 70, entraînant une rupture profonde de l'économie traditionnelle et la réduction drastique des populations nomades qui passaient de $75 \%$ de la population au début des années 70 à moins de $20 \%$ aujourd'hui. Or ces populations nomades, grâce à l'élevage, exploitaient des ressources rares et dispersées dans tout l'espace saharien : l'incapacité où elles furent, de par la longueur et la répétition rapide des sécheresses, de reconstituer leur cheptel entraîna des bouleversements non seulement dans la structure de l'économie mauritanienne, mais également dans la répartition spatiale des populations et, plus important que tout le reste sans doute, des transformations sociétales aux effets incalculables en termes d'équilibre des communautés en Mauritanie et au delà, dans la région. L'effondrement de l'économie nomade allait en effet d'abord déverser massivement sur les zones utiles ou proches de la distribution des aides internationales par l'État, une grande part de ces populations en voie de paupérisation rapide. D'où la croissance prodigieuse des villes mauritaniennes : Nouakchott qui comptait quelques milliers d'habitants au moment de l'Indépendance, en concentre aujourd'hui plus de 600 000, soit près d'un tiers de la population nationale. A la faveur des investissements qui étaient effectués sur la fleuve et par suite de l'évolution de la législation foncière qui libérait juridiquement les terres de leur statut coutumier et dépouillait ainsi les communautés indigènes de leurs monopoles juridiques traditionnels, une bonne partie de ces anciens nomades vint aussi chercher ressources dans la vallée du fleuve. Si parmi eux on pouvait trouver des Peuls (les Peuls nomades, dits Peuls jeeri par opposition aux Peuls agriculteurs, dits Peuls waalo), l'essentiel appartenait à l'ethnie maure. Le rétrécissement de l'espace rural 
mauritanien sur quelques zones promises à une relative prospérité accrut donc la pression démographique sur le fleuve et exacerba dans cette région antérieurement à dominante démographique soudanophone la concurrence interethnique qui depuis l'indépendance s'était concentrée sur la question du contrôle du pouvoir central et la question culturelle (les soudanophones récusant une arabisation forcée) et la déplaçait ainsi sur le terrain directement économique. Cette surpression démographique s'accompagnait d'une complexification de la question des rapports interethniques, du fait des transformations sociétales que devait subir la société maure et qui feraient qu'une fraction de cette société serait lancée par l'autre fraction à l'assaut de la région fluviale, apparaissant comme l'instrument d'une arabisation nouvelle, par occupation territoriale directe, et sans doute réveillant le souvenir de la progression méridionale des anciens émirats maures qui jadis avaient refoulé les négro-africains de l'autre côté du fleuve. Disons d'abord deux mots de la structure de la société maure elle-même et voyons ensuite comment le rapport au territoire interne devait affecter l'équilibre local et régional, entraînant - comme dans le cas Touareg, une internationalisation du conflit. Entre la structuration de la société maure et de la société touarègue une correspondance assez étroite a pu être établie que globalement résume assez bien le tableau suivant: Correspondances dans la structure hiérarchique maure et touarègue (d'après Djibo M. Hamani, 1989, p. 303) ${ }^{43}$

2 Société maure ordre Société touarègue Hassan 1 Imajaghan Zwâia 2 Ineslem Aznâga 3 Imghad Harratin 4 Ighawallan et Idarfan Ma'allemîn et Iggâwan 5 Inadan et Ikanawan Abîd 6 Iklan

Se trouvaient donc en haut les nobles 1) gens de sabre (guerriers) ou 2) du Livre, puis venaient en 3 les "vassaux" ou tributaires. Ces trois groupes formaient les hommes libres. Puis arrivaient les dépendants hors tribus (puisque rattachés directement à une des catégories précédentes): en 4) les affranchis, en 5) les "artisans" et en 6) les esclaves. Pour ce qui est de la société maure, les deux premiers niveaux sont composés de populations revendiquant une origine arabe (1) ou plutôt arabe (2), tandis que le niveau 3 s'attribue une origine berbère, les niveaux inférieurs étant eux composés de populations noires ${ }^{44}$, comme chez les Touareg. Chaque strate de la société maure traduit les superpositions successives des nouveaux occupants du territoire qui, au lieu d'éradiquer les populations plus anciennement installées, leur ont attribué des statuts sociaux qui établissaient un partage hiérarchique des fonctions et des bénéfices du nouveau mode de production ainsi élaboré, la catégorie des esclaves se renouvelant alors grâce aux razzias. Comme on voit, non seulement existait une interpénétration spatiale stratifiée interethnique, mais à l'intérieur des ensembles ethniques eux-mêmes se déployait un système social emboîté, qui fut à sa manière et à travers les temps un système d'intégration efficace : ainsi les noirs qui furent jadis réduits en esclavage ont oublié leur culture négro-africaine d'origine et adopté la culture des maîtres maures, au point d'émarger politiquement de leur côté (idem chez les Touareg). Si l'esclavage a été aboli officiellement en Mauritanie pour la dernière fois en 1982, il n'empêche que la société maure demeure très hiérarchique, sinon de droit du moins de mentalité, ce qui a toujours des effets sociaux considérables; et chacun se positionne encore aujourd'hui en fonction de sa catégorie d'origine: on ne manque pas de rappeler de tel ou tel ministre qu'il est "hartani" ou "forgeron"! Chez les soudanophones, une hiérarchisation forte existe également avec tout en haut les torodos. La sévérité de la sécheresse ayant donc définitivement déséquilibré l'économie et la société nomades, 
l'une des composantes de cette société, les harratin, à savoir désormais tous les anciens esclaves, donc tous les noirs arabisés, qui constituaient la main d'œuvre des nomades maures, se sont trouvés dans une situation de complet abandon : leur ultime libération juridique qui fut donc réitérée en 1982 était d'une certaine façon l'aveu de l'impuissance de leurs maîtres à désormais pouvoir les employer et donc les nourrir. Aussi en 1975 vit-on apparaître le mouvement d'émancipation des affranchis El Hor, qui traduit l'émergence d'une prise de conscience de groupe de ces déshérités. Et ces déshérités, de serviteurs ils pourraient devenir une classe dangereuse pour leurs anciens maîtres s'il leur prenait l'idée de faire valoir leur force de frappe démographique: probablement entre 35 et $40 \%$ de la population nationale, soit certainement beaucoup plus que les Maures blancs (les beydan), et de contracter alliance avec les soudanophones dont ils dépassent sans doute aussi, dans des proportions difficilement appréciables (faute de publication des deux derniers recensements), le nombre global, devenant ainsi, ce qui est une nouveauté radicale de la vie politico-sociale mauritanienne, un nouveau facteur d'instabilité. Les Maures, sous la présidence militaire du colonel ould Taya (1984-1992) ${ }^{45}{ }^{46}$, ont compris ce danger et permis l'accession symbolique au pouvoir de quelques ministres harratin. Et surtout, afin de détourner le cours d'une possible colère populaire, ils ont cherché un espace économique auquel les associer. Les aménagements agricoles de la vallée du fleuve offraient une double opportunité : une nouvelle loi foncière favorisant l'appropriation privative de ces terres, un coup double serait tenté en permettant aux investisseurs maures d'acquérir des terres dans cette région et aux harratin de trouver du travail. Par ce même mouvement, les Maures accroissaient leur emprise économique et faisaient des Harratin des alliés dans le combat qui ainsi était lancé contre les soudanophones occupants de la vallée. Percevant dans le déploiement insidieux de cette politique une nouvelle avancée des Maures vers le sud et l'analysant d'abord comme la conséquence de la monopolisation du pouvoir central, des éléments de l'élite soudanophone (surtout en fait des Haalpularen) créèrent dès 1983, un mouvement de résistance : le Front de Libération Africain de Mauritanie (FLAM), ayant son siège à Dakar; en 1986 certains éléments publièrent le Manifeste du négro-mauritanien opprimé qui valut à ses auteurs la prison où trois d'entre eux périrent des traitements administrés; en 1987 des officiers haalpularen tentaient un coup d'État réprimé de façon expéditive et sanglante; la montée inexorable de cette tension entre les deux communautés aboutit aux événements sanglants d'avril-mai 1989 (plusieurs centaines de morts) qui internationalisèrent un conflit au départ purement intérieur. La tension sur la frontière avec le Sénégal prit prétexte d'un incident entre pasteurs noirs des deux rives du fleuve pour dégénérer d'abord au Sénégal, puis en Mauritanie, en affrontements réglés entre Maures et Sénégalais, puis en Mauritanie même entre Maures et Harratin d'un côté et soudanophones nationaux de l'autre. Devant les violences incontrôlables, des milliers de Maures du Sénégal furent "rapatriés" en Mauritanie, tous les Sénégalais durent faire de même. Mais ce qui fut plus grave et qui mobilisa le Sénégal et d'autres pays sud-sahariens, quand les massacres ou menaces de massacre ne suffisaient pas, ce furent les expulsions massives de soudanophones nationaux, voire de Peuls arabisés, vers le Sénégal où ils furent placés dans des camps qu'ils occupent toujours aujourd'hui. On assistait ainsi des deux côtés du fleuve à un mouvement de population que certains observateurs soupçonnèrent de programmation préalable du côté maure tellement il fut mené de façon systématique et efficace. Le Sénégal se vida de ses commerçants maures (dont des Harratin), la Mauritanie de ses 
Sénégalais (mécaniciens, pêcheurs) et de plus d'une centaine de milliers de soudanophones nationaux : une véritable "purification ethnique" avant celle de Bosnie ou du Rwanda. On assista à la rupture des relations diplomatiques et à la réactivation officielle de la revendication par Senghor de rectification de la frontière entre les deux pays de manière que la rive droite du fleuve fut incluse dans le territoire sénégalais. En définitive, là où avait existé une transterritorialité relative de l'économie et de la société, allait-on assister à un renforcement de l'État-territoire comme résultat d'une montée des nationalismes? Peut-on comparer la rébellion / résistance Touareg au Niger et au Mali avec la question mauritanienne et mauritano-sénégalaise ? Disons d'emblée qu'il y a une différence fondamentale : les deux grands partis qui s'affrontent en Mauritanie débordant surtout vers le Sénégal ressortent chacun d'une certaine façon de deux univers assez clairement identifiés, l'ensemble arabe pour les maures, l'ensemble négro-africain pour les soudanophones, et chaque ensemble a ses supporters en quelque sorte "naturels", en simplifiant d'une part les États arabes et d'autre part les États noirs; pour les Touareg la situation n'est pas totalement symétrique ni de la situation des Maures puisqu'ils n'ont pas le pouvoir, ni, comme minorité, de la situation des soudanophones de Mauritanie, puisqu'en tant que berbères et berbérophones ils peuvent certes bénéficier de sympathies dans les communautés berbères d'Algérie et $\mathrm{du}$ Maroc, mais d'une part les liens intercommunautaires sont très distendus et ces sympathies assez symboliques; et surtout il n'existe nulle part d'État berbère, situation comparable à celle des Kurdes comme l'énonce lui-même un des représentants de la résistance touarègue ${ }^{47}{ }^{48} 49$, Mano Dayak (1992). Incontestablement la grande faiblesse de la résistance touarègue, c'est l'absence à l'extérieur de toute territorialité-refuge "naturelle", ce qui fait que, devant toujours dépendre de la bonne volonté d'un État étranger en cas de difficulté intérieure majeure, ils sont davantage exposés que d'autres à la manipulation comme prix de l'aide accordée. On peut ainsi comprendre que pour les Touareg comme pour les Kurdes aujourd'hui, ou les Juifs dans l'esprit et à l'époque de Herzl (affaire Dreyfus, pogroms en Russie), la question territoriale put être jugée capitale pour la survie comme peuple, du moins tant que d'autres modèles d'État alternatifs par rapport à l'État-nation-territoire n'avaient pas cours sur la scène africaine. Le second aspect de la question touarègue vient de ce que dans le passé les Touareg ont été dominateurs par rapport aux populations au milieu desquelles ils vivent toujours, mais dont ils sont devenus désormais les compatriotes sans privilèges, se plaignant plutôt que celles-ci aient pris une revanche en les marginalisant: les soudanophones mauritaniens ne furent eux jamais des maitres par rapport aux Maures. Affirmant parfois une lointaine origine commune avec les Maures, les Touareg sont même directement associés aux Maures maliens dans leur révolte récente au Mali. Leur image est ainsi brouillée régionalement : ni noirs, ni arabes, en des régions où, en définitive, il vaut mieux être l'un ou l'autre. Anciens maîtres devenus victimes, leur révolte si elle ravive et se nourrit de la mémoire de la résistance héroïque et longtemps victorieuse contre la pénétration française, réveille aussi pour les négro-africains des souvenirs moins heureux de domination : "Un peuple essentiellement guerrier, particulièrement mobile, suffisamment imprévisible et à l'esprit naturellement indépendant. Bref un peuple plutôt insaisissable, profondément jaloux de son indépendance mais, dans le même temps, curieusement prompt à ne pas respecter celle des autres", selon le jugement de l'homme politique et universitaire nigérien ${ }^{50} \mathrm{~A}$. Salifou. La question touarègue telle qu'elle est posée aujourd'hui remonte en fait aux indépendances. Les chefs touareg 
d'alors n'avaient pas vu venir la vague d'émancipation des peuples colonisés (en quoi ils ne se différenciaient d'ailleurs pas des notables maures). Lorsqu'il fut question (1956) de la création de l'Organisation Commune des Régions Sahariennes (OCRS) qui eut permis à la France d'envisager la division de la colonie algérienne entre le nord et le Sahara qu'elle eut gardé sous couvert de cette organisation, les Touareg adhérèrent, y voyant une opportunité de regroupement où ils auraient constitué un élément démographique important et qui en tout cas eut réuni dans un seul ensemble la plus grande partie d'entre eux. Quel que soit le jugement qu'on puisse porter sur cette tentative que rappelent aujourd'hui avec nostalgie des résistants touareg comme Mano Dayak et que Djibo M. Hamani ${ }^{51}$ qualifie de "réponse apportée par les milieux les plus rétrogrades de la métropole aux aspirations des peuples colonisés", la décolonisation ne prit pas cette tournure, les négociateurs algériens refusant toute négociation sur la cession du Sahara. Les Touareg furent alors contre leur gré disloqués entre plusieurs États. Certes on peut discuter pour savoir si jamais ils avaient été unis politiquement: "Il n'y a jamais eu un monde touareg uni, ni politiquement ni économiquement; la frontière actuelle du Niger avec l'Algérie correspond à la limite des zones d'influence du sultanat d'Ayar (Aïr) et de l'Amanokal de l'Ahagar ; la frontière entre le département de Tawa et le Mali correspond à la limite entre l'Amanokalat des Kel Nan et celui des Iwillemmeden Kel Ataran; les Kel Geres et Iteseyan s'étaient séparés des Kel Ayar à la suite de guerres; les Ibarkoreyan ont été expulsés de l'Ayar au XVIIe siècle après des guerres. Ces divisions sont nées des contradictions internes aux sociétés touareg du Nord et de l'Ouest et il est inutile de leur chercher un bouc émissaire. Il y eut, au cours de l'histoire, plus de guerres entre les Touareg qu'entre ces derniers et d'autres peuples" écrit Djibo M. Hamani52(1993) qui ajoute aussitôt : "Il est vrai que la remarque vaut également pour les Hausa, les Zarma, les Tubu et d'autres." Cependant on ne peut toujours invoquer le passé pour interdire l'avenir. En tout cas la situation actuelle des Touareg découle directement des choix qui furent faits par la France dans sa politique de décolonisation et auxquels fait écho le Programme-cadre de la Résistance : "Le Niger est une entreprise franco-française pilotée depuis Paris. En effet, ce n'est un secret pour personne, la France n'a pu devenir une puissance nucléaire que grâce à l'uranium de nos terres. C'est ce dernier et toutes les autres richesses que recèle notre Sahara qui nous ont valu tous nos malheurs, entre autres celui d'avoir été rattachés au Niger" (p. 4). En tout cas dès le départ, les Touareg récusèrent les divers rattachements territoriaux auxquels ils furent cependant contraints de se soumettre, au péril de leur mode de vie économique qui naturellement, pour eux qui étaient surtout nomades, faisait écologiquement fi des frontières et conséquemment au péril de leur survie en tant que société spécifique. Et ce que l'Empire français avait encore permis, ces mouvements entre les différentes entités administratives coloniales, l'accession à l'indépendance séparée des composantes de l'empire devait le rendre plus difficile, et à terme l'interdire. Comme en Mauritanie, les mêmes causes produisant les mêmes effets, une sécheresse durable et répétée $(1965,1967,1973,1984)$ devait ajouter aux difficultés nées de l'enfermement relatif dans des espaces réduits. Ce fut le début de l'éclatement en trois groupes de l'ancienne communauté nomade : tandis qu'une partie continuait à pratiquer le mode de vie traditionnel grâce d'une certaine manière à la dépopulation qui allait s'opérer par des départs massifs, une deuxième partie rejoignit les villes du Niger ou du Mali et une troisième, surtout des jeunes, s'expatrierait ${ }^{53}{ }^{54}$. Faute de pouvoir réagir en groupe, la communauté touarègue dans un premier temps éclatait donc. Nous ne sommes pas dans le cas de figure des deux communautés mauritaniennes 
qui ont, elles, tendance à se renforcer continuement. Mais alors que le règlement de la question touarègue semblait devoir ainsi se régler dans cette région par l'éclatement de cette communauté, sa dispersion, sa "normalisation" dans les espaces étatiques internes, voici qu'éclata au grand jour une rébellion armée au Niger (mai 1990) qui s'étendit aussitôt au Mali (juin 1990). Cette rébellion se présente elle même comme un mouvement de résistance qui, à l'instar du mouvement érythréen par exemple, s'en prend directement à des États africains auxquels ils font le reproche de colonisation interne. On verra cependant que les Touareg ne demandent pas nécessairement une totale indépendance, ce qui est un élément particulièrement intéressant quant à la question de l'évolution de la stato-teritorialité. Rappelons brièvement ces événements et tentons d'en saisir la genèse dans ses différentes dimensions, au delà des éléments structurels de départ. Si en Mauritanie ce sont des problèmes frontaliers qui déclenchèrent des événements en chaîne sans à proprement parler de mouvement armé (en dehors des escarmouches des deux armées régulières, mauritanienne et sénégalaise, sur le fleuve et de quelques éclats limités du FLAM), dans l'affaire touarègue on a assisté à deux événements qui d'emblée ont pris une tournure armée, signifiés par deux noms de lieu : Tchin-Tabaraden au Niger les 6-7 mai 1990, Menaka au Mali le 29 juin 1990. Tchin-Tabaraden, ville du nord-nigérien, connut une attaque armée touarègue: deux gendarmes nigériens furent tués, des armes prises par les assaillants, les représailles de l'armée gouvernementale furent lourdes. Les assaillants touareg qui le purent s'enfuirent au Mali, le président Moussa Traoré, solidaire du président nigérien et surtout soucieux de préserver son pays de l'importation de la crise, procéda à l'arrestation de rebelles nigériens : le 26 juin la prison de Menaka où les Touareg nigériens avaient été incarcérés fut violemment attaquée et les Touareg maliens se lançaient à leur tour dans la révolte. Très rapidement l'affaire prit de l'ampleur et amena les deux gouvernements à chercher des solutions au delà de la simple répression, d'autant plus que des mouvements populaires par ailleurs allaient ouvrir ces pays à une transition démocratique ( $\$ 4)$. Comment expliquer, non la fièvre subite, puisqu'on sait que le feu couvait depuis l'indépendance, mais l'ampleur des événements et l'inquiétude réelle des gouvernants? En fait la dislocation à laquelle la société touarègue semblait condamnée de par sa dispersion en trois groupes : ruraux maintenus, urbains sous ou mal-intégrés et exilés, ne s'est pas produite. Face à la désespérance de la société nomade et à la collaboration à la marge de la société urbanisée, le groupe des exilés, ces jeunes gens qui avaient dû quitter le Niger et le Mali parce que chômeurs, ishumar en français targuisé ${ }^{55}$, allait concevoir une stratégie de résistance qui s'avèrerait redoutable le moment venu. Avec l'arrivée du colonel Kadhafi au pouvoir en Libye, ce pays devint à la fois attractif économiquement et idéologiquement. Selon le Programme-cadre de la Résistance (rédigé en 1994), en partant en Libye (et à un degré moindre en Algérie) les jeunes répondaient "à un appel du sein" et d'ajouter: "Ayant coïncidé avec le démarrage des programmes révolutionnaires mis en place pour le développement de ce pays, nous avons pu apprécier de visu ce que doivent être les rapports entre les pouvoirs et leurs administrés. Et là, le miroir nous renvoya l'ampleur et la profondeur des injustices que nous subissons chez nous" (p. 6). En septembre 1980, Kadhafi lança un appel solennel aux Touareg leur offrant de les accueillir dans "leur" pays : la Libye ${ }^{56}$. Une fois rendus sur place par dizaine de milliers, ces ishumar devinrent des ishumar kalachnikov, ils rejoignirent la Légion islamique de Kadhafi, servirent sur tous les fronts les desseins du colonel libyen: Sahara occidental, Tchad (par exemple lors de la bataille de Faya- 
Largeau en 1987), Liban aux côtés des Palestiniens ${ }^{57}$. Tchad, Niger, Mali : reprenant sans doute les babouches des émirs de la Sénoussya ${ }^{58}$, Kadhafi poursuivait une même politique, la création d'un grand État libyo-sahélien et les ishumar furent parmi les instruments de cette politique. Et la politique du colonel ne fut pas seulement active à l'égard des exilés puisque, par deux fois, la main du colonel fut soupçonnée derrière des tentatives de coup d'État au Niger du temps du genéral Seyni Kountché : à chaque fois (1976, 1983) les autorités nigériennes accusèrent un Libyen, un certain Liman Chaafi, qui en 1976 se réfugia en Mauritanie et en 1983 s'était associé à un targui transfuge du cabinet du ministère de la défense nigérien et réfugié en Libye, Khamed Moussa ${ }^{59}$. La sécheresse de 1983-84 ajouterait aux répressions des Touareg consécutives à la tentative de coup d'Etat de 1983, et de nouvelles vagues d'éxilés rejoindraient les camps à l'étranger, en Libye mais aussi en Algérie. A la fin des années 1980, le révolutionnarisme de Kadhafi pour de nombreuses raisons tiédit, au point qu'au moment de la Seconde Guerre du Golfe il ne rejoignit même pas le camp de Saddam Hussein. Son attitude vis à vis des ishumar (pluriel d'ashamur) se transforma, son soutien à l'idée de "république des Touareg" disparut pratiquement, mais les ishumar n'entendirent pas pour autant renoncer. Si Kadhafi ne fut en aucune façon accusé par les autorités maliennes et nigériennes d'avoir été à l'origine des insurrections de 1990, même un analyste aussi manifestement favorable à la cause touarègue que $D$. Rosenberg ${ }^{60}$ est obligé de reconnaitre que sa politique passée "a joué un rôle décisif dans le déclenchement des événements actuels en contribuant à la structuration à la fois intellectuelle et matérielle du mouvement d'autodétermination touareg". De nouveaux événements allaient faire évoluer la donne et permettre un sursaut des ishumar. Le général Kountché que Kadhafi et eux-mêmes avaient tant combattu mourut en 1987, son successeur le général Ali Saïbou chercha à changer l'image du régime et devait se résoudre à ouvrir un processus démocratique. Avec Kadhafi, il inaugura de bonnes relations et en mars 1989, lors d'un voyage en Libye, il offrit l'amnistie aux exilés nigériens. Ceux-ci posèrent quelques conditions: des mesures d'équité pour leur communauté dans les allocations de l'État et un statut d'autoadministration pour le nord du Niger où ils sont démographiquement le plus implanté. L'Algérie se trouvant dans l'impossibilité de continuer à entretenir les camps de réfugiés touareg sur son sol, décida en 1988 d'en renvoyer plusieurs milliers; de nouvelles mesures d'expulsion furent prises en 1989, une aide du FIDA pour leur réinsertion aussi bien au Mali qu'au Niger fut obtenue qui devait rendre possible le transfert pacifique de ces populations déplacées ; 20 à 25000 réfugiés furent rassemblés en janvier 1990 à In-Guezzam près de la frontière algéro-nigérienne, les Maliens les refusèrent comme Nigériens et réciproquement. 18000 furent à la fin acceptés par le Niger et parqués précisément près de Tchin-Tabaraden. Parmi eux les ishumar ${ }^{61}$. L'aide internationale parvint difficilement aux camps, l'accusation fut portée de détournement par les autorités nationales et locales, des troubles se multiplièrent dans les camps de rapatriés ${ }^{62}$. On sait la suite, les ishumar reprirent les hostilités contre le pouvoir d'État, cette fois non plus depuis le territoire libyen, mais sur leur terre même. Le bilan des morts de l'été 1990 témoigne de la violence des combats : au Mali plus de 600, moitié militaires réguliers, moitié Touaregs ${ }^{63}$; au Niger officiellement environ 70 morts, environ 600 d'après Amnesty International, un millier selon les Touareg64. Les luttes pour le territoire sont humainement coûteuses... Pour conclure cette partie, disons en tout cas que si la géopolitique est "l'étude des relations qui existent entre la conduite d'une politique de puissance portée sur le plan international et le cadre géographique dans lequel elle 
s'exerce" ${ }^{65}$. Si dans certaines phases de l'histoire récente des États régionaux on peut incontestablement voir intervenir des "politiques de puissance" (Libye, France, Irak, Maroc, Algérie, Arabie Séoudite essentiellement), force est de constater que s'agissant aussi bien des soudanophones mauritaniens que des Touareg le facteur sociétal est l'ingrédient fondamental, déclencheur des crises, confirmant ainsi les analyses de Bertrand Badie et M.-Cl. Smouts ${ }^{66}$ sur "l'irruption des sociétés" dans les relations internationales d'aujourd'hui et de l'impossible maintien de l'opposition classique entre l'interne et l'externe ${ }^{67}$. Issues différenciées des crises ou la territorialité entre renforcement et recomposition. Ces crises régionales - dont il est inutile de retracer ici le détail des péripéties ultérieures - ont depuis 1990 évolué. Et d'abord la menace de guerre civile a ,pour l'heure, disparu. Mais la question du croisement entre statoterritorialité et ethno-territorialité qui fut l'enjeu des deux crises examinées, avec dans chaque cas leurs spécificités, a-t-elle été résolue? Ou bien l'un des camps aurait-il remporté une victoire complète sur un adversaire? Et celui-ci, à moins d'homogénéisation socio-territoriale totale par ethnocide ou déportations massives, serait-il définitivement hors-jeu? Ce qui est intéressant dans l'étude de l'issue (actuelle) de ces crises, c'est la différence relative des voies suivies sur les plans interne et international d'une part, la différence relative par ailleurs des réponses apportées quant à l'évolution régionale de la stato-territorialité. En bref, on pourrait dire que là où les soudanophones mauritaniens sont sortis plutôt victimes des compromis interétatiques menés sous influence internationale et où l'on a assisté à un renforcement relatif de la stato-territorialité classique. Dans le cas de la question touareg est intervenue une novation relative, dont les Touareg, au moins sur le papier et à condition qu'il y ait une suite effective sur le terrain, sont plutôt bénéficiaires : la promotion d'une autre stato-territorialité que celle de l'État-nation-territoire, plus en rapport peut-être avec des évolutions en cours ailleurs. L'issue de crise n'a pas suivi en effet les mêmes voies pour la Mauritanie, le Sénégal et pour les deux autres États sahéliens. Dans le premier cas, la surdétermination du conflit interne par les enjeux internationaux était considérable. La crise mauritanienne prenait ses racines dans l'échec de la double appartenance identitaire, arabe et négro-africaine, qui eut dû demeurer constante dans la politique nationale de par le projet fondateur de l'État indépendant : la Mauritanie avait été définie alors, par Moktar ould Daddah, comme un pays-pont entre le monde noir et le monde arabe, comme un pays-frontière ${ }^{68}$. Avec le temps, l'élément maure a joué surtout et très majoritairement la carte du nationalisme arabe, particulièrement sensible au Baath d'obédience irakienne ${ }^{69}$, au point que si le gouvernement n'adoptait pas officiellement cette idéologie (voire s'en défendait en mettant de temps à autre quelques militants en prison), il avait fait de la Mauritanie un pays allié de Saddam Hussein, plus manifestement encore après la fin de la guerre IranIrak (août 1988). De leur côté les soudanophones, face (selon eux) à la dérive arabe des alliances de l'État et à la politique d'arabisation suivie à l'intérieur condamnant à terme leur communauté à l'assimilation à l'ethnie maure (la beydanisation), comme les Harratin, réactivèrent un nationalisme négro-africain endémique depuis l'Indépendance. En Mauritanie, ce sont donc deux nationalismes qui s'affrontent, le nationalisme arabe partisan d'une beydanisation totale du pays à la fois par la langue et par le rattachement au système régional arabe, le nationalisme négro-africain (impulsé surtout par les Haalpularen) voulant le maintien de la double identité de la Mauritanie et un partage équitable du pouvoir politique, économique et culturel, voire un cadre fédéral, ce qui fut envisagé et aussitôt repoussé par Moktar ould Daddah déjà dans les 
années 60. La période d'exacerbation de la crise correspond d'ailleurs au plus fort de la montée des nationalismes, après la tentative de coup d'Etat de 1987 à l'instigation d'officiers haalpularen et l'implication plus forte régionalement de l'Irak, que renforcerait en 1990 l'appel de Saddam Hussein à la solidarité qui reçut un écho enthousiaste de la part de l'élément maure : le pari aveugle sur une victoire du leader irakien conduirait au massacre gratuit de centaines de militaires soudanophones en décembre 1990. Le Sénégal, qui avait en 1989 ses problèmes politiques intérieurs auxquels la crise mauritanienne apporta un dérivatif, sut alors jouer de ses relations privilégiées avec la France mais aussi avec le Maroc, pays certes partenaire de la Mauritanie dans l'Unité du Maghreb arabe, mais aussi pays à la mémoire longue (refus de la création de la Mauritanie, intérêts opportunément convergents puis structurellement divergents sur la question du Sahara occidental) et à la politique constante : une volonté de maintien de son influence historique en Afrique noire. La défaite de Saddam Hussein ayant déjoué les paris de l'élite maure, la crise indissociablement internationale et interne du pays eut emporté le régime si celui-ci ne s'était montré apte à des manœuvres rapides, à la fois sur le plan international et sur le plan interne. Après que d'autres États eussent en vain tenté une médiation entre les deux belligérants, la France, qui à l'apogée de la crise de 1989-1990 avait été taxée de complicité évidente avec le Sénégal ennemi, fut admise comme intermédiaire et amena les deux gouvernements au dialogue puis au compromis, chacun retirant son aide aux opposants intérieurs (FLAM mauritanien soutenu par le Sénégal, nationalistes casamançais en lutte contre Dakar soutenus par la Mauritanie). Par le même mouvement, le pouvoir, qui quelques mois auparavant était internationalement condamné pour atteinte caractérisée aux Droits de l'Homme, annonça l'ouverture d'un processus démocratique, qu'il conduirait effectivement en 1992 et 1993 avec un degré de réussite non négligeable. L'ouverture inopinée de ce processus désarçonna les opposants au régime et conduisit le FLAM à renoncer officiellement dès 1992 au recours à la violence. Les négociations pour la relance des actions communes (Mali, Mauritanie, Sénégal) de l'office de mise en valeur de la vallée du Sénégal (OMVVS) reprirent et reçurent l'assistance financières des grands organismes internationaux et de puissances européennes. Mais à la crise ethno-territoriale à forte charge économique aucune réponse réelle ne fut apportée, ni idée fédéraliste, ni idée régionaliste, pas de réforme non plus sur la question foncière. Les soudanophones expulsés n'ont retrouvé leurs terres que dans des proportions limitées, quand ils ne sont pas maintenus dans des camps au Sénégal, six ans après les événements et trois ans après l'ouverture du processus démocratique. L'acceptation de la reprise de la coopération dans le cadre de l'OMVVS prouve que l'État sénégalais, qui fut très engagé dans la crise auprès des soudanophones, a renoncé de fait à les soutenir dans la recherche d'une équité ethnique en Mauritanie même. Dakar aurait-il préféré que ses "frères de race" soutinssent jusqu'au bout sa volonté de rectification de la frontière de façon qu'ils rejoignissent ainsi le Sénégal, et augmentant du même coup son territoire ? Aurait-il été déçu qu'ils ne manifestassent aucun attrait pour cette solution, proclamant même vouloir rester mauritaniens? La fraternité du Sénégal avec le Maroc dans la crise ne laissait-elle pas croire que ces deux États pourraient s'entendre pour un partage de la Mauritanie ? Hypothèses pas invraisemblables mais incontrôlables, et craintes au moins feintes du côté mauritanien. Au lendemain de la seconde Guerre du Golfe, régionalement, la France restait la seule puissance vraiment influente auprès des deux États et les accords qui furent passés sous son égide visèrent surtout à rétablir dans la 
région l'ordre des États : d'après A. Salifou qui parle d'expérience directe ${ }^{70}$, ce serait la DGSE française dirigé par le préfet Silberzahn et le même homme sur le terrain, le colonel Vié, qui auraient eu à charge de conduire le règlement des dossiers mauritanosénégalais et touareg! Même si la conditionnalité démocratique fut invoquée par les différents bailleurs de fonds occidentaux (mais pas arabes) qui reprirent la route de Nouakchott après la guerre du Golfe, la démocratie exigée était de fait très formelle, car en dehors de la considération sur la nature de la règle du jeu politique, point d'intérêt ne fut accordé aux solutions des problèmes qui avaient conduit le pays à sa dernière crise. D'une certaine manière l'accord interétatique entérinait les avantages ethnoterritoriaux "acquis" lors de la cessation des hostilités, la sécurité importait plus que la solution des problèmes de société, une certaine "raison d'État internationale" avait primé. On ne chercha pas de solution novatrice à la question stato-territoriale: si la coopération interétatique dans la mise en valeur du fleuve connut un nouveau départ, la frontière, elle, perdit sa porosité, sortit ethniquement plus étanchéifiée de l'épreuve. Les 70000 mauritaniens soudanophones toujours réfugiés au Sénégal se contenterontils indéfiniment de la raison des États? Ira-t-on vers une nouvelle "irruption des sociétés" dans l'ordre régional ? La comparaison de l'issue de cette crise avec celle de la question touarègue est instructive, encore qu'il faille sans doute ici distinguer plus finement entre les évolutions (en cours) au Mali et au Niger. Les acteurs de la crise mauritano-sénégalaise étaient deux États et deux ensembles ethniques, dont l'un, les Maures, se confondait pratiquement avec l'État mauritanien. Cette situation est radicalement différente des crises malienne et nigérienne. Ici les États sont confrontés en même temps et indépendamment non seulement à une même crise, mais aux mêmes adversaires "civils" : des deux côtés de la frontière c'est un même ensemble ethnique, même subdivisé, voire divisé, qui d'un même mouvement entre en insurrection. D'emblée, le phénomène eut un caractère transnational, et surtout l'adversaire des malien ou nigérien n'était pas un autre État, par allégeance ethnique interposée (comme ce fut partiellement, en termes d'alliance au moins, possible si Kadhafi avait continué à intervenir activement dans la région) ou directement comme dans la crise mauritano-sénégalaise. Les données du problème sont objectivement différentes et la solution à la crise ne pouvait pas être prise tout à fait par le même biais, une négociation interétatique classique: si l'État n'est pas en mesure d'éradiquer l'insurrection ou ne le veut pas pour des raisons idéologiques (choix démocratiques) ou d'image internationale, l'interlocuteur qu'il peut avoir ne peut être que le mouvement insurrectionnel lui-même. Quelques considérations géopoliques allaient permettre l'internationalisation de la question, dans des conditions différentes de la crise mauritano-sénégalaise. Notons d'abord que les Touareg sont majoritairement concentrés (à la fois du point de vue de l'implantation géographique et proportionnellement à toutes les autres ethnies locales) dans le nord du Mali et du Niger, sont ainsi ethno-terrritorialement mieux définissables que d'autres ethnies; or ne les enclavant pas dans les espaces étatiques respectifs (comme ce serait le cas pour d'autres ethnies), cette concentratrion (relative) à la périphérie septentrionale rend possible une autonomisation de leur territoire, considéré par les Touareg comme une réalité politique précoloniale dont le mythe fut à sa façon (et ses fins) exploité par le colonel Kadhafi auprès des ishumar . Les Touareg sont ensuite et corrélativement à ce qui vient d'être dit, dans une situation qui rappelle celle des Kurdes, et singulièrement les Touareg nigériens par comparaison avec les Kurdes irakiens : on pourrait en effet comparer Arlit et ses mines d'uranium situées au Nord du Niger en "pays touareg", à 
Kirkouk et ses champs pétrolifères situé en "pays kurde". Or l'uranium est un enjeu économique majeur pour l'État nigérien et une ressource stratégique pour la France en particulier. Selon des statistiques de 1985, le Niger détenait officiellement $11,2 \%$ des réserves (ressources déjà inventoriées) mondiales d'uranium, soit l'équivalent de 55 années de production, et $13,6 \%$ des ressources (réserves supputées), soit l'équivalent de 146 années d'exploitation ${ }^{71}$. Le "pays touareg" n'est donc pas la vallée du fleuve sénégal dont l'enjeu économique n'est que local, ni une "simple" réserve anthropologique (les "hommes bleus") pour safaris touristiques : l'intérêt exotique des medias occidentaux, intelligemment mobilisés par quelques leaders touareg, cachait l'intérêt pour une zone économique sensible, la mise en place rapide à Paris d'une cellule propre pour traiter la crise montrant le sérieux avec lequel la question fut d'emblée prise. L'internationalisation, non du conflit (il ne déborda pas quant à lui des zones touarègues maliennes ou nigériennes), mais de son traitement d'issue de crise, se fit en deux temps. Il y eut d'abord une première phase où les responsables officiels tentèrent de mobiliser les ressources ordinaires de la diplomatie, et où son règlement fut envisagé comme un simple problème de police certes internationale, mais de police quand même. A l'initiative du président malien Moussa Traoré, les États limitrophes (Algérie, Libye, Mali et Niger) tentèrent de trouver une réponse qui, dans l'esprit du chef de l'État malien comme du chef de l'État nigérien, devait faire appel à la solidarité des États. Kadhafi - dont on sait pourtant lla tiédeur de l'appui apporté désormais aux nationalistes touareg - arriva à la rencontre de Djanet (sud-algérien) les 8-9 septembre 1990 en grande tenue touarègue et avec le président Bendjedid condamna les pratiques discriminatoires des États sahéliens à l'égard de leurs ressortissants touareg. Différentes résolutions interétatiques furent alors prises, dont celle d'enterrer définitivement toute accusation contre la Libye, de créer un comité quadripartite permanent devant procéder à une surveillance étroite des mouvements migratoires et frontaliers, ainsi que de réunir régulièrement les quatre ministres des affaires étrangères afin de renforcer les bonnes relations entre les États. La conférence de Djanet n'eut pas de suite. Dans une deuxième phase, de par des volontés indépendantes mais convergentes des Touareg et des gouvernants du Niger et du Mali, l'évolution de la crise allait prendre un tour différent : la solution de problèmes internes et que les États sahéliens voulaient continuer à traiter comme tels, se fit par l'appel non plus à des arrangements interétatiques, inefficaces du fait que les combattants touareg, faute d'appui, s'étaient totalement autonomisés par rapport au "protecteur" libyen, mais à des médiations étrangères entre les parties internes en conflit. Dans le cas mauritanosénégalais, la France certes facilita la rencontre (fin 1991-1992) entre les deux États, mais jamais elle n'amena le gouvernement mauritanien à négocier avec les négronationalistes; dans le cas de la crise touarègue, les intermédiaires étrangers sollicités (surtout Algérie, Libye, France) allaient conduire à une même table de négociation les représentants ès-qualité de la résistance touarègue et ceux des gouvernements nationaux. Une internationalisation réelle donc, mais une internationalisation particulière. Le "facilitateur" algérien que sollicitèrent les Maliens ne relevait pas, au grand dam des Touareg, du ministère des affaires étrangères, mais du ministère de l'intérieur, comme si la question touarègue était prise par les Algériens en rapport avec les problèmes qu'elle avait occasionnés sur leur propre territoire et devait être traitée comme une question de police des frontières ; et l'on a déjà vu que dans le cas nigérien où la France joua un rôle moteur, la médiation fut concrètement assurée moins par le Ministère des affaires étrangères que par la DGSE. Ainsi si c'est au terme de rencontres 
patronnées par des États étrangers que la sortie de crise s'opéra, les Touareg n'obtinrent pas pour autant une reconnaissance qui leur eut donné le statut de mouvement de libération. En fait les précautions diplomatiques prises par les puissances extérieures visaient à ne pas déstabiliser les États malien et nigérien ni à remettre en question leur intégrité territoriale (difficilement soutenable en particulier pour les intérêts français dans le cas nigérien). Ne pouvant véritablement jouer sur une indépendance que ne soutiendraient pas (Algérie) ou plus (Libye) les pays riverains, les Touareg maliens, en dehors de quelques fractions jusqu'au-boutistes, réclamèrent seulement une organisation fédéraliste de l'État. Le 6 janvier 1991, avec la médiation algérienne, intervinrent les accords de Tamanrasset entre le gouvernement malien et les Touareg maliens menés par le réprésentant du Mouvement populaire de l'Azawad (nom donné au pays touareg) et du Front islamique arabe de l'Azawad (front maure): parmi les mesures envisagées, sous réserve de ratificaton par le parlement national, figurait non un fédéralisme mais une régionalisation très poussée pour l'Azawad. Après quelques reculs sur ces accords, notamment à la suite du renversement du président Traoré par le lieutenant-colonel Touré en avril $1991^{72}$, une nouvelle crise se déclencha qui provoqua un nouvel exil de Touareg et de Maures maliens et une forte mobilisation internationale (deux médiateurs supplémentaires furent envoyés au Mali, l'un mauritanien : Ahmed Baba Miske, et l'autre français : E. Pisani). Mais le renversement du président Traoré devait permettre l'ouverture d'un processus démocratique au Mali et le débat public de la question touarègue. Après que l'Algérie eut favorisé sur son sol une rencontre entre les différentes composantes de la résistance touarègue à El-Goléa, les grandes lignes d'un accord, obtenu en présence du médiateur algérien et du président malien en personne, fut conclu entre l'État et les Touareg à Mopti les 16-18 décembre 1991 et le 11 avril 1992 était officiellement signé par les différentes parties le Pacte national ${ }^{73}$. Ce Pacte est particulièrement intéressant à étudier et peut préfigurer des évolutions intéressantes de la territorialité. Il propose en effet des solutions originales: le fédéralisme d'une certaine façon règle les rapports des populations à l'intérieur d'un État, les solutions adoptées par les négociateurs prennent davantage en compte les spécificités du monde touareg et promeuvent une forme de régionalisation à différentes échelles, du niveau local au niveau régional-interne et même au niveau sahélo-transnational. Je citerai seulement quelques articles de ce Pacte à l'appui de mon affirmation. Le Titre III porte reconnaissance d'un "statut particuler du Nord du Mali", l'Azawad des Touareg. Sont créées dans ce Nord malien des Assemblées locales, régionales et interrégionales habilitées à traiter des questions de développement économique, social et culturel. Au niveau interrégional (chapitre I de ce Titre III) il est écrit dans l'article 18: "Dans le respect de l'unité de l'État et de la Nation du Mali et dans le but de favoriser une politique de développement dans une partie du territoire national partageant une très forte similitude de paramètres géographiques, climatiques, socio-économiques et culturels au profit des populations concernées et au bénéfice de la République du Mali, il sera institué une assemblée inter-régionale au niveau des Régions du Nord du Mali"; et dans l'article 23, de préciser que parmi les compétences de cette assemblée figure la contribution "à l'animation et la promotion du développement transfrontalier avec les pays voisins", formule qui sera répétée pour l'assemblée de niveau local. La formule sera adaptée pour l'assemblée de niveau régional d'une manière qui mérite attention: "Dans le respect de la souveraineté nationale et des engagements de l'État, l'Assemblée de la Région a compétence pour promouvoir une politique de développement transfrontalier et un programme de 
coopération et d'échanges avec des institutions similaires de pays voisins" (chapitre II, article 32) : certes vu d'Europe ceci peut paraittre aujourd'hui ordinaire, mais en Afrique (et surtout en Afrique ex-française) il y a là une nouvelle manière d'envisager, et le territoire et la souveraineté nationale qui est notable. Il est vrai que pour que de pareilles attributions puissent devenir réalité, il faudrait que les pays voisins adoptent une organisation administrative similaire, et en particulier le Niger. Si celui-ci adoptait les mêmes dispositions, alors pourrait-on dans le cas touareg, auquel cette solution est réservée au Mali, assister au développement d'une autre territorialité que la statoterritorialité, et elle aurait d'autant plus de chance de développement et serait une alternative valable pour d'autres ethnies corsetées par des États Jacobins qu'une nouvelle organisation régionale des États africains verrait le jour, sinon la création d'un espace touareg officiel transnational mais de niveau infra-étatique à nouveau risquerait de déboucher sur une nouvelle revendication de territorialité d'État, un État touareg cette fois. En ses articles 58 et 59, le Pacte énonce d'ailleurs clairement les conditions de réussite de cette nouvelle politique: “Convaincu que la solidarité et l'unité nationales trouvent leur prolongement naturel dans la solidarité et l'unité africaines, le Gouvernement de la République du Mali a réitéré sa détermination à soutenir sont action de réconciliation et de paix nationales par un effort pour la promotion de la coopération et du développement sous-régionaux. A cet égard le Gouvernement de la République du Mali redoublera d'action pour la relance de la coopération entre les Etats et les peuples de l'Organisation des Etats Sahariens complément indispensable des autres Organisations sous-régionales auxquels appartient la République du Mali". Au Niger, la solution de la question touarègue ne semble pas, pour le moment, avoir fait d'aussi grands pas dans le sens de la novation dans le domaine territorial. Certes le Niger fut bien lui-aussi à son tour touché par la vague de démocratisation qui déferlait alors sur l'Afrique. La Conférence souveraine nationale qui siégea du 29 juillet au 3 novembre 1991 mit en place les institutions d'une transition qui, le président Ali Saïbou demeurant en fonction, durerait jusqu'aux élections présidentielles pluralistes d'avril 1993. Un débat très dur eut lieu sur le problème touareg lors de la conférence nationale, l'armée fut clairement mise en cause pour Tchin-Tabaraden notamment, les militaires furent convoqués devant des commissions ad hoc, avec la participation active de Touareg, un processus de réconciliation nationale fut entamé, tandis que fin 1991 était créé le Front de Libération de l'Azawad et de l'Aïr (FLAA). La France joua un rôle particulièrement actif dans la quête d'un rapprochement en particulier entre le gouvernement de la transition et la résistance touarègue. Mais le règlement de la question fut plus long qu'au Mali, sans doute parce qu'ici les enjeux sont plus grands (de par l'importance numérique des Touareg et stratégique de l'uranium). Un accord de principe est enfin intervenu entre "le Gouvernement de la République du Niger et la Coordination de la Résistance Armée" (CRA, représentée par Mano Dayak) lors de la conférence tenue à Ouagadougou les 15-17 février 1994, "en concertation avec les délégations de l'Algérie, du Burkina Faso, de la France et du Mali" (communiqué de presse officiel) ${ }^{74}$.Dans le programme de la CRA qui fut présentée lors de cette rencontre, l'idée fédérale est maintenue.comme solution adéquate à la crise. Pour les élections législatives (anticipées) qui sont annoncées (1995), l'Union pour la Démocratie et le Progrès Social (UDPS-Amana), parti touareg créé en 1991 mais qui avait été interdit de participation aux élections de 1993, réitère dans son programme actuel la proposition de fédération. D'autres solutions que le fédéralisme ont été envisagées, parmi lesquelles un "partage du pouvoir entre les différentes nationalités", des 
institutions à la libanaise qui organiseraient les attributions des responsabilités par ethnie. Comme l'objecte un membre de l'UDPS-Amana, "même si les institutions de l'Etat doivent réfléter les diversités communautaires du pays, l'unité nationale ne saurait se réduire à une simple arithmétique ethnique et/ou régionale" (A. Anamégui, 1995). A l'inverse les objections contre le fédéralisme, outre qu'elles s'alimentent des difficultés que rencontre la Nigeria fédéraliste voisine, se fondent moins sur une analyse de la répartition géographique des Touareg (les données empruntées à André Salifou réunies dans la carte $n^{\circ} 2$ montrent que $84 \%$ des Touareg se trouvent dans les départements du Nord: Tillaberi, Tahoua et Agadès) que sur le fait que même là où leur concentration est forte, vivent également d'autres ethnies (ainsi la ville d'Agadès est à dominante haoussa, et pas seulement depuis des temps récents) et que se poserait alors la question du droit des autres minorités ethniques présentes dans ce qui serait l'Etat fédéré touareg. Cet Etat devrait-il être vraiment touareg ou citoyen? Un fédéralisme d'Etats ethniques serait-il viable? La purification ethnique ne frappe-t-elle pas aujourd'hui un peu à toutes les portes des nationalismes renaissants? Ce n'est pas la stato-territorialité en soi qui pose problème, mais le national-territorialisme, la territorialité dans des espaces qui ne sont pas homogènes ethniquement, et ceci est valable aussi bien en Afrique que par exemple en Bosnie, au Kosovo ou en Macédoine ${ }^{75}$ Carte administrative du Niger et répartition des Touareg par départements (d'après A. Salifou 1993, 6, 107) ${ }^{76}$ Touareg / département (1988)

Tahoua 202833 Zinder 36688 Tillabéri 155315 Dosso 9170 Agadès 114020 Maradi 37 515 Diffa 1513

5 Mais pour qu'il y ait des territorialités fluides (dans le passé pré-colonial elles étaient plutôt floues que fluides), faudrait-il qu'on retrouve des identités plus souples, comme aussi elles l'étaient sans doute à une époque d'exacerbation moindre des querelles ethniques, qui résultent en bonne partie de l'échec (relatif) de l'Etat post-colonial en ces zones. La réflexion sur les limites du droit à la différence d'A. Salifou ${ }^{77}$, est à cet égard intéressante : "Il faut que les unes et les autres (ethnies), et particulièrement les Touareg en rébellions, sachent que leur spécificité n'est pas intemporelle, et qu'elle doit d'autant plus s'adapter à son temps que le monde lui-même est en perpétuel mouvement". Et à l'égard de l'Etat, sa réflexion rejoint cette des rédacteurs du Pacte malien: "L'Etat quant à lui doit se convaincre de ce que sa recherche, aussi obstinée que vaine, de l'unité nationale doit désormais cesser de se poursuivre dans le cadre étroit d'une micro-nation, pour s'inscrire dans le contexte plus large de la sous-région". Conclusions Ainsi assiste-t-on dans cette partie de l'Afrique saharo-sahélienne à une prise de conscience progressive de l'inadaptation ethno-territoriale du modèle de l'Etat-nation qui fut imposé-adopté au lendemain des décolonisations. Si la solution de la crise mauritano-sénégalaise est demeurée encore classique (y compris s'agissant du montage de la coopération pour la mise en valeur de la vallée du fleuve, alors qu'elle eut pu être l'occasion de renouveler, d'adapter d'anciennes territorialités), la solution de la crise touarègue a mis en œuvre un autre type de règlement. D. Rosenberg ${ }^{78}$ écrit à propos du règlement de la question au Mali : "La présence et la signature de l'Algérie ne peuvent être réduites à une formelle et tierce médiation nécessaire au rapprochement des positions et à la garantie de l'accord : elles se conforment à la tradition régionale, selon laquelle la question touarègue, par essence transnationale, doit faire l'objet d'un règlement concerté entre les Etats concernés". Si ainsi pendant un temps ils ont voulu assurer la police par la défense de l'internationale des Etats, aujourd'hui ces mêmes Etats sont obligés, pour mieux assurer leur pouvoir, de reconnaître la relativité de leur 
souveraineté, ce que signifie fortement le fait que les Etats du Niger et du Mali aient consenti à résoudre la question du rapport à leurs nationaux en recourant volontairement à de tierces puissances. L'Occident se cherche : "Nous sommes dans une période de flou, où il ne suffit pas de dire 'Etat', 'souveraineté', 'politique' pour redonner vie à ces réalités. Mais il n'y a pas d'autre principe qui s'y substitue (...). Nous avons retrouvé aujourd'hui la polarité Gemeinschaft-Gesellschaft. Et les grandes institutions qui étaient censées faire la médiation, avoir un certain contenu affectif et en même temps une certaine rationalité bureaucratique sont en crise, qu'il s'agisse des Etats nationaux ou des communautés supra-nationales"79. En Afrique l'OUA qui a tant fait de son principe de l'uti possidetis un dogme, est touchée par cette relative dévaluation du territoire et de la souveraineté et l'on remarquera que dans le règlement des crises sahéliennes examinées (sauf au début et en vain pour la crise mauritano-sénégalaise) elle n'a joué aucun rôle et n'est sans doute pas adaptée au règlement de crises à dimension internationale dès lors que les partis en présence ne sont plus seulement étatiques. L'Occident se cherche, l'Afrique aussi.

\section{NOTES}

1. Marcel Merle, La crise du Golfe et le nouvel ordre international, Economica, Paris, 1991.

2. Christian Schricke, «L'unification allemande », in .Annuaire Français de Droit International XXXVI, Ed. du CNRS, Paris, 1990, pp. 47-87.

3. Raymond Goy, «L'indépendance de l'Erythrée », in Annuaire Français de Droit International XXXIX, Ed. du CNRS, Paris, 1993, pp. 337-356.] et le règlement de la question cambodgienne[[Paul Isoart, « La difficile paix au Cambodge », in Annuaire Français de Droit International XXXVI, Ed. du CNRS, Paris, pp. 249-265.

4. Jean-Marc Sorel, « La Somalie et les Nations Unies », in Annuaire Français de Droit International XXXVIII, Ed. du CNRS, Paris, 1992, pp. 61-88.

5. Zaki Laidi, 1994, Un monde privé de sens, Fayard, Paris, 333 p.

6. «Le défi saharien », in P.R. Baduel (dir.), Enjeux sahariens, Editions du CNRS, Paris, 1984, 442 pp., 13-27.

7. Ce qui renvoie pour le Maroc en particulier à l'opposition classique entre Bled Maghzen et Bled Siba qui justifiait l'appellation de "pacification" donnée par les Français à leur opération d'éradication de la résistance à leur occupation, en faisant passer leur action comme une contribution à la soumission au sultan de régions présentées comme ne dépendant de lui que nominalement, le Bled Siba, pays de la dissidence.

8. André Martel, Les confins saharo-tripolitains de la Tunisie (1881-1911), PUF, Paris, 1965, 2 t.. Saïd Sayagh, Le Maghzen, les tribus-frontières et la France de 1873 à 1902, Thèse de 3e cycle, Université de Provence, Aix-en-Provence, 1982. Olivier Vergniot, " Tindouf, un point d'équivoque (1912-1934) », in P. R. Baduel éd., Désert et montagne au Maghreb. Hommage à Jean Dresch, Revue de l'Occident musulman et de la Méditerranée, pp.41-42, Edisud, Aix-en-Provence, 1986, 422 p., 119-135. 
9. Chaouki Serghini, Le Maroc et la Mauritanie dans les relations internationales, Thèse d'Etat en droit, Nice, 1982, 728 p.

10. Bernard Lanne, Tchad-Libye, la querelle des frontières, Karthala, Paris, 251 p., et André Martel, La Libye, 1835-1990. Essai de géopolitique historique, PUF, Paris, 1982, $256 \mathrm{p}$.

11. «Mauritanie 1945-1990 ou l'État face à la nation », in P. R. Baduel (dir.), Mauritanie entre arabité et africanité, Revue du Monde musulman et de la Méditerranée 54 (nº́cial), Edisud, Aix-en-Provence, 1990, 200 p., pp. 11-51. La brève guerre de février 1995 entre le Pérou et le Guatémala montre que l'Amérique latine n'est pas à l'abri de la réactivation des revendications frontalières.

12. «La production de l'espace national au Maghreb », in P.R. Baduel (dir.), États, territoires et terroirs au Maghreb, Editions du CNRS, Paris, 1985, 421 p, 3-47.

13. Marie-Christine Aquarone, Les frontières du refus : six séparatismes africains, $E d$. du CNRS, Paris, 1987, 142 p.

14. Mais le Maroc, seul avec la Somalie, avait récusé dès le départ ce principe de la charte de l'OUA.

15. Roland Marchand, « La Corne de l'Afrique », in Politique africaine $n^{\circ} 50$, Karthala, Paris, 1993, pp. 2-87.

16. Roland Marchand, « La Corne de l'Afrique », in Politique africaine $n^{\circ} 50$, Karthala, Paris, 1993, pp. 2-87.

17. R. Goy, op. cit., pp. 355-56.

18. P.R. Baduel, op. cit..

19. R. Marchand, op. cit.

20. Christian Delmet, "Construction de l'Etat et conflits de nationalismes au Soudan ", in P.R. Baduel (dir.), Etat moderne, nationalismes et islamismes, Revue du Monde Musulman et de la Méditerranée, 68-69, Edisud, Aix-en-Provence, 1994, pp.87-98.

21. «Rébellion » est le langage employé par les dirigeants de l'État nigérien ou malien à l'égard d'un mouvement que les Touareg qualifient eux de « résistance ».

22. « Un conflit tectonique », in La Croix du 6 septembre 1989.

23. René Otayek René, Le radicalisme islamique au sud du Sahara. Daw'a, arabisation et critique de l'Occident, Karthala, Paris, 1994, 264 p.

24. Hassner, op. cit.. pp. 25-26.

25. Kenneth. N. Walts, Man, the State and War. A theoretical analysis, Columbia University Press, New York and London, 1959, 263 p. pp. 12-14.

26. Les descriptions de ces sociétés données par certains ethnologues les ont amenés à parler de « sociétés de castes ». Cette qualification fait problème, surtout si on établit une comparaison rigoureuse avec la société à propos de laquelle elle a été conceptualisée, la société indienne : ici le critère fondateur en Inde de la hiérarchie, l'opposition pur/impur, ne joue pas en particulier dans les catégories supérieures, les "guerriers" occupent la première place et non les "marabouts", les critères de classification étant assez simples, le nombre des catégories est incomparablement moindre qu'en Inde, etc... (cf. L. Dumont : Homo Hiérarchicus, R. Deliège, Le système des castes). Comme il n'y a pas lieu d'en débattre davantage ici pour les besoins du présent exposé, je parlerai simplement de "société hiérarchique".

27. Lire Front de Libération Africaine de Mauritanie (section Europe), , Livre blanc sur la situation des noirs en Mauritanie. Radioscopie d'un apartheid méconnu, 1989, 284 p. 
28. Ces données sont tirées de P. Boilley; A. Salifou, donne un chiffre encore moindre pour les Touareg nigériens : 557054 âmes, soit 7,6 \% de la population totale du pays en 1988.

29. Coordination de la Résistance Armée, 1994, Programme-cadre de la Résistance, 50 p. 30. Salifou André, La question touarègue au Niger, Karthala, Paris, 1993, 203p. p.141. 31. Sur les Peuls, qu'on a qualifiés d'éternels juifs errants de l'Afrique de l'Ouest (Ch. Sentoir : «Les Peuls « refusés »: Les Peuls mauritaniens réfugiés au Sénégal (département de Matam) », in Cahiers des Sciences humaines de l'Orstom, Paris, 1990 pp. 577-603), on peut lire l'incontournable ensemble d'études dirigé par R. Botte et J. Schmitz , «L'Archipel peul », Cahiers d'études africaines,XXXIV (1-3), Paris, 1994.

32. Salifou, op. cit., p. 197.

33. Stewart Charles $C$., « Une interprétation du conflit mauritano-sénégalais », in P.R. Baduel (dir.), Mauritanie entre arabité et africanité, Revue du Monde musulman et de la Méditerranée 54, Edisud, Aix-en-Provence, 1990, 200 p., pp. 161-170.

34. Salifou, op. cit., p., 104.

35. Roy Olivier, « Ethnies et politique en Asie centrale », in O. Roy (dir.), Des ethnies aux nations en Asie centrale, Revue du Monde Musulman et de la Méditerranée 59-60, Edisud, Aix-en-Provence, 1992, 288 p., pp. 17-36.

36. Bayart Jean-François, L'Etat en Afrique. La politique du ventre, Fayard, Paris, 1989, 439 p.. J-L. Amselle écrit de son côté clairement dans Logiques métisses. Anthropologie de l'identité en Afrique et ailleurs : "L'identité se définit comme un écart ou comme une différence. C'est également l'oubli des conditions de production du social et du politique qui fonde l'identité ou l'ethnicité. (...) Les faits d'ethnicité ressortissent à une lecture essentiellement politique ». En Europe l'idée de nation ne s'est pas contruite autrement (cf B. Anderson, Imagined Communities. Reflections on the origin and spread of Nationalism, Verso editions and NLB, Londres, 1983) et s'agissant de la division entre Germaniques et Français l'ouvrage de C. R. Brühl : Naissance de deux peuples. Français et Allemands (IXe-XIe siècles), Fayard, Paris, 1995. 388 p. ; vient d'en apporter une nouvelle démontration, à un moment où la montée de l'idéal politique européen amène à déconstruire les mythes fondateurs de l'ancien ordre stato-national. 37. Cauneille A., Les Chaanba. Evolution de la tribu durant l'administration française, Editions du CNRS, Paris, 1968, 319 p.

38. Baduel Pierre Robert, 1980, Société et émigration temporaire au Nefzaoua (SudTunisien), Editions du CNRS, Paris, 144 p.

39. Sentoir, Ch., « Le repli Peul en Mauritanie à l'ouest de l'Assaba. L'évolution du peuplement peul sur la rive droite du fleuve Sénégal (départements de Kaédi, Maghama, Monguel et Mbout) ", in Cahiers d'Outre-Mer 46 (182), Bordeaux-Talence, 1993, pp. 131-151. ; et Leservoisier Olivier, La question foncière en Mauritanie. Terres et pouvoirs dans la région du Gorgol, L'Harmattan, Paris, 1994, 351 p.

40. Retaillé Denis, « La conception nomade de la ville », in Jean Bisson (dir.), Le nomade, l'Oasis et la Ville, URBAMA, Tours, 1989, pp. 21-35.

41. Selon Ch. Sentoir : "Le conflit mauritano-sénégalais : la genèse. Le cas des peuls de la haute vallée du Sénégal » in Cahiers des Sciences humaines de l'Orstom, 26 / 4, Paris, 1990, pp. 553-576, dans le temps il arrivait même que, dans le cadre de l'économie de prédation qui sévissait alors, Maures et négro-africains - en l'occurence des Peuls (la partie des négro-africains il est vrai la plus ouverte à l'arabisation, sans pour autant jamais fusionner avec les Maures chez qui la négritude était synomyme de dépendance, voire d'esclavage) - passassent alliance pour se livrer à un même razzou, mais avec 
partage différencié du butin : ainsi les seconds razziaient-ils les troupeaux quand les premiers s'emparaient des autres biens et des hommes.

42. Marchesin Philippe, Tribus, ethnies et pouvoir en Mauritanie, Karthala, Paris, 1992, $437 \mathrm{p}$.

43. Hamani Djibo Mallam, Au carrefour du Soudan et de la Berbérie : le sultanat touareg de l'Ayar, Institut de Recherches en Sciences Humaines, Etudes nigériennes $n^{\circ} 55$, Niamey, 1989, $521 \mathrm{p}$.

44. Bonte Pierre, Conte Edouard, Hames Constant et Ould Cheikh Abdel Wedoud, Al Ansâb, la quête des origines. Anthropologie historique de la société tribale arabe, Editions de la Maison des Sciences de l'Homme, Paris, 1991, 260 p. pp., 141-144.

45. En 1992 le colonel Ould Taya s'est présenté aux premières élections présidentielles pluralistes et libres de la Mauritanie indépendante et a été élu président de la République.

46. Dayak Mano, Touareg. La tragédie, Lattès, Paris, 1992, 221 p.

47. Dayak Mano, Touareg. La tragédie, Lattès, Paris, 1992, 221 p.

48. Salifou, op. cit.., p 21.

49. Salifou, op. cit.., p 21.

50. Salifou, op. cit.., p 21.

51. Hamani, Djibo Mallam, «Une gigantesque falsification de l'histoire », in Niyya ${ }^{\circ} 4$

(avril 1994), Niamey, pp 5-8.

52. Ibid. Hamani, op. cit.,.

53. Dayak, Mano, op. cit., p. 70.

54. Claudot-Hawad Hélène (dir.), Touaregs, exil et résitance, Revue du Monde Musulman et de la Méditerranée 57 (nº spécial), Edisud, Aix-en-Provence, 1991, 199 p.

55. Claudot-Hawad Hélène (dir.), Touaregs, exil et résitance, Revue du Monde Musulman et de la Méditerranée 57 (nº spécial), Edisud, Aix-en-Provence, 1991, 199 p. 56. Rosenberg Dominique, «Le peuple touareg. Du silence à l'autodétermination », in Revue belge de droit international 2, Ed. Bruylant, Bruxelles, 1992, pp.5-39.

57. Dayak, Mano, op. cit. , pp. 69-86.

58. Triaud Jean Louis, 1987, Tchad 1900-1902, une guerre franco-libyenne oubliée ? Une confrérie musulmane, la sanusiyya, face à la France, L'Harmattan, Paris, 203 p.

59. Maniaque de l'annexion territoriale, en 1976 Kadhafi avait publié une carte qui, comme pour la bande d'Aouzou (Tchad), s'attribuait d'office $20000 \mathrm{~km} 2 \mathrm{du}$ territoire nigérien . Salifou, op. cit., pp., 42-44.

60. Rosenberg, op. cit., p 15.

61. Dayak, op. cit., $\mathrm{p} 86$.

62. Rosenberg, op. cit., p. 19.

63. Rosenberg, op. cit. p. 20.

64. Dayak, op. cit., p. 90.

65. Gallois Pierre M., Géopolitique, les voies de la puissance, Préface de René-Jean Dupuy, Plon/Fondation pour les Etudes de Défense Nationale, Paris, 1990, 474 p.

66. Badie Bertrand et Smouts Marie-Claude, 1992, Le retournement du monde.

Sociologie de la scène internationale, Presses de la FNSP/Dalloz, Paris, $249 \mathrm{p}$.

67. Baduel, « La Mauritanie dans l'ordre international », in P. Bonte et H. Guillaume (dir.), Mauritanie. Un tournant démocratique ?, Politique africaine 55, Karthala, Paris, pp. 11-19.

68. Baduel, op. cit.,. 
69. Baduel, Pierre-Robert, « La difficile sortie d'un régime autoritaire. Mauritanie 1990-1992 », in Revue du Monde Musulman et de la Méditerranée 63-64, Edisud, Aix-enProvence, 1993, pp. 225-243.

70. Salifou, op. cit. p.103, 172, 178.

71. Giraud André et Boy de la Tour Xavier, Géopolitique du pétrole et du gaz, Editions Technip, Paris, 1987, 418 p. p.46.

72. Moussa Traoré était un ami du président mauritanien Ould Taya. Son renversement fut un événement qui, joint à l'échec irakien, contribua sans doute à la réflexion du chef de l'Etat mauritanien et faciliterait l'acceptation d'une ouverture démocratique à Nouakchott.

73. Texte intégral in P. Boilley 1994, pp. 905-920. Boilley Pierre, « Les droits d'un peuple ", in Le Monde des débats 10 (juillet-août), Paris, 1993 pp. 27-28.

74. On remarquera l'absence de la Libye parmi les signataires.

75. Roux Michel, 1992, Les albanais en Yougoslavie. Minorité nationale, territoire et développement, Editions de la Maison des Sciences de l'Homme, Paris, 546 p.

76. Salifou, op. cit., p. 107.

77. Salifou, op. cit., p. 202.

78. Rosenberg, op. cit., p. 26.

79. Hassner, op. cit. pp. 377-78.

INDEX

Mots-clés : Etat-nation, territoire(s) et territorialité

Index géographique : Afrique subsaharienne, Maghreb 